\title{
Therapy Standards in HER2-Positive Breast Cancer
}

\author{
Chair: \\ Oleg Gluz
}

\author{
Participants: Cornelia Liedtke ${ }^{b}$ Frederik Marméc $^{c}$ Marc Thill $^{d}$ \\ a West German Study Group, Brustzentrum Niederrhein, Mönchengladbach, Germany; \\ ${ }^{b}$ Konservative Tumortherapie und Studienzentrale, Brustzentrum, Klinik für Frauenheilkunde und Geburtshilfe, \\ Universitätsklinikum Schleswig-Holstein / Campus Lübeck, Lübeck, Germany; \\ c Sektion Translationale Gynäkologische Onkologie, Nationales Centrum für Tumorerkrankungen und Universitätsfrauenklinik Heidelberg, \\ Heidelberg, Germany;

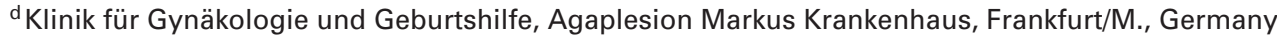

\section{Question 1. Dual Blockade for HER2-Positive Breast Cancer - How to Select Patients for Neoadjuvant Chemotherapy in Combination with Trastuzumab / Pertuzumab?}

Liedtke: Dual blockade including a combination regimen with trastuzumab and pertuzumab has been shown to increase pathological complete remission ( $\mathrm{pCR}$ ) rates in comparison to chemotherapy and trastuzumab alone. However, despite its favorable toxicity profile, the addition of pertuzumab in all patients with HER2positive breast cancer in the neoadjuvant setting carries the risk of both overtreatment and increased treatment costs. Therefore, there is an urgent need to identify subgroups of patients who carry an overall prognosis that is favorable enough to allow exclusion of pertuzumab from their treatment regimen. Predictive factors relevant to this aspect still need to be defined. In the NeoSpere trial, for instance, negative hormone receptor status was significantly and positively associated with an increased chance of pCR. Unless there will be valid parameters in the future, strategies to decide for or against the addition of pertuzumab may rely on baseline prognostic factors (such as tumor size or nodal status) and predictive markers (such as hormone receptor status).

Marmé: Several strategies can be used to select patients for dual blockade strategies. Today, in routine clinical practice stage of the disease to assess the patient's risk is probably the most commonly used method to select patients for dual blockade, additional factors, such as age, hormone receptor status and concomitant diseases, also play a role. Patients who fulfill the inclusion criteria of the APT trial [1] are likely to fare well with trastuzumab plus chemotherapy alone and dual blockade is likely to be overtreatment as long as survival data of the APHINITY trial are still pending. In the near future, efforts should be taken to validate promising biomarkers, such as tumor infiltrating lymphocytes, estrogen receptor-positive $(\mathrm{ER}+)$ status and PI3K mutations. In addition, early response evaluation should also be assessed as a possible stratification factor.

Thill: Adding pertuzumab to trastuzumab in the neoadjuvant setting (NEOSPHERE [2] and TRYPHAENA [3] study) led to a significantly higher pCR rates. Again, the benefit for HER2-positive patients was improved. The survival data of the NEOSPHERE study that were presented at ASCO 2015 showed an increased disease free survival (DFS) for patients who achieved a pCR and consequently another evidence for a translation of an increased pCR into a higher DFS [4]. In my institution patients with a tumor size of $\geq 2 \mathrm{~cm}$ - along with the inclusion criteria of the NEOSPHERE study - are treated with the combination of trastuzumab and pertuzumab in addition to chemotherapy. In case of tumors $<2 \mathrm{~cm}$ with unfavorable characteristics (i.e. Ki67 of $>35 \%$ or $\mathrm{pL} 1$ or $\mathrm{pV} 1$ ) the dual blockade in addition to chemotherapy is also preferred.

\section{Question 2. Less Is More - Which De-Escalation Strategies Are Promising for Which Patients with HER2-Positive Breast Cancer?}

Liedtke: In my opinion, the most promising aspect is the reduction in chemotherapy intensity through the use of dual blockade strategies. It is imaginable that the addition of pertuzumab may allow for either developing an anthracycline-free chemotherapy backbone or even a chemotherapy-free treatment schedule. Studies, such as the ADAPT HR-/HER2+ trial [5], explore these strategies.

\section{KARGER}

Fax +497614520714

\section{() 2016 S. Karger GmbH, Freiburg}

1661-3791/16/0112-0148\$39.50/0 
Patients are randomized to a 12 -week therapy with either trastuzumab/pertuzumab alone or trastuzumab/pertuzumab in combination with paclitaxel. Yet, patients are recommended to complete standard taxane (and anthracycline) chemotherapy as postneoadjuvant therapy - however, it seems possible, that in case of a) demonstration of solid treatment efficacy and b) identification of response predictors, this recommendation might be alleviated in the future.

Marmé: The thought of a chemotherapy-free treatment for a subset of HER2-positive breast cancer patients is provoking and promising. However, we need strategies to identify suitable patients (e.g. lymphocyte predominant breast cancers) or reliable methods for early response evaluation. These may include PET imaging (like in the AVATAXHER trial) and could help to implement such a 'dual blockade without chemotherapy' strategy or other strategies that spare our patients chemotherapy and toxicity (e.g., dual blockade plus less toxic chemotherapies, such as vinorelbine, eribuline, or T-DM1). Patients with a poor response to these regimens could then go on to receive salvage chemotherapy plus targeted therapy. Optimal duration of these de-escalated regimens will also have to be evaluated.

Thill: The study by Tolaney et al. [1] examined trastuzumab plus single weekly paclitaxel in early HER2 positive breast cancer and showed excellent data with a 3-year-survival free from invasive disease of $98,7 \%$ and a 3 -year-recurrence free survival of $99.2 \%$. The survival was better than expected. $62.2 \%$ of the patients had tumors of $\leq 1 \mathrm{~cm}$ and $10.1 \%$ an age of $>70$ years. Therefore, these results offer an option to treat smaller or more favorable HER2positive tumors or maybe older patients with a single chemotherapy instead of a combination chemotherapy.

In metastatic disease, the recent published study by Dang et al. [6]showed excellent survival data for the first-line treatment with trastuzumab + pertuzumab and paclitaxel instead of the combination with docetaxel. This might be a combination with a more favorable toxicity.

\section{Question 3. What Is the Optimal 'Place' for T-DM1 in the 'HER2 Space'?}

Liedtke: The most important aspects with regard to T-DM1 are its solid efficacy and, most importantly, good tolerability. In the ADAPT HR+/HER2+ trial we have seen pCR rates of close to $50 \%$ in association with an excellent toxicity profile. Similar to concepts relevant to dual blockade as mentioned above, inclusion of T-DM1 into the neoadjuvant setting may allow for reduced treatment toxicity without the cost of reduced treatment efficacy.
Marmé: Currently, T-DM1 is the standard second-line regimen for HER2-positive breast cancer and standard first-line treatment in patients relapsing early after adjuvant chemotherapy + trastuzumab (and pertuzumab). However, T-DM1, due to its very favorable toxicity profile, is an interesting agent in the neoadjuvant setting and has demonstrated convincing activity in the triple-positive arm of the ADAPT trial. Individualizing therapy, e.g. by giving 4-6 cycles T-DM1 as neoadjuvant therapy, and offering additional adjuvant therapy, e.g. including trastuzumab plus pertuzumab and chemotherapy, to those not achieving pCR with a high Neo-Bioscore could be attractive strategies.

Thill: To date, T-DM1 is the compound of choice in second-line treatment of HER2-positive metastatic breast cancer and, based on the retrospective analysis of the EMILIA study, in the treatment of brain metastases of HER2 positive breast cancer. It is conceivable that due to better tolerabilty and lower toxicity T-DM1 will replace trastuzumab and chemotherapy in metastatic breast cancer as shown in the MARIANNE trial [7]. It will not replace the dual blockade in combination with docetaxel in first-line treatment [8], unless that is not feasible due to patient's comorbidities. However, further studies could show the advantage of T-DM1 as a more tolerable HER2-directed approach in early breast cancer.

\section{Participants}

PD Dr. med. Cornelia Liedtke

Konservative Tumortherapie und Studienzentrale, Brustzentrum Klinik für Frauenheilkunde und Geburtshilfe Universitätsklinikum Schleswig-Holstein / Campus Lübeck Ratzeburger Allee 160, 23538 Lübeck, Germany cornelia.liedtke@uksh.de

PD Dr. med. Frederik Marmé

Leitung Sektion Translationale Gynäkologische Onkologie Nationales Centrum für Tumorerkrankungen und Universitätsfrauenklinik Heidelberg Im Neuenheimer Feld 460, 69120 Heidelberg, Germany Frederik.Marme@med.uni-heidelberg.de

PD Dr. med. Marc Thill

Klinik für Gynäkologie und Geburtshilfe Agaplesion Markus Krankenhaus Wilhelm-Epstein-Straße 4, 60431 Frankfurt/M., Germany marc.thill@fdk.info 


\section{References}

1 Tolaney SM, Barry WT, Dang CT, et al: Adjuvant paclitaxel and trastuzumab for node-negative, HER2 positive breast cancer. N Engl J Med 2015;37:134-341.

2 Gianni L, Pienkowski T, Im YH, et al: Efficacy and safety of neoadjuvant pertuzumab and trastuzumab in women with locally advanced, inflammatory, or early HER2-positive breast cancer (NeoSphere): a randomised multicentre, open-label, phase 2 trial. Lancet Oncol 2012;13:25-32.

3 Schneeweiss A, Chia S, Hickish T, et al: Pertuzumab plus trastuzumab in combination with standard neoadjuvant anthracycline-containing and anthracyclinefree chemotherapy regimens in patients with HER2positive early breast cancer: a randomized phase II cardiac safety study (TRYPHAENA). Ann Oncol 2013; 24:2278-2284.
4 Gianni L, Pienkowski T, Im YH, et al: Five-year analysis of the phase II NeoSphere trial evaluating four cycles of neoadjuvant docetaxel (D) and/or trastuzumab (T) and/or pertuzumab (P). ASCO Meeting Abstracts 2015;33:505.

5 Harbeck N, Gluz O, Christgen M, et al: Final analysis of WSG-ADAPT HER2+/HR+ phase II trial: Efficacy, safety, and predictive markers for 12-weeks of neoadjuvant TDM1 with or without endocrine therapy versus trastuzumab+endocrine therapy in HER2-positive hormone-receptor-positive early breast cancer. Cancer Research 2016;76: abstr S5-03.
6 Dang C, Iyengar N, Datko F, et al: Phase II study of paclitaxel given once per week along with trastuzumab and pertuzumab in patients with human epidermal growth factor receptor 2-positive metastatic breast cancer. J Clin Oncol 2015;33:442-447.

7 Ellis PA, Barrios CH, Eiermann W, et al: Phase III, randomized study of trastuzumab emtansine (T-DM1) $\{+/-\}$ pertuzumab $(\mathrm{P})$ vs trastuzumab + taxane $(\mathrm{HT})$ for first-line treatment of HER2-positive MBC: primary results from the MARIANNE study. ASCO Meeting Abstracts 2015;33:507.

8 Verma S, Miles D, Gianni L, et al: Trastuzumab emtansine for HER2-positive advanced breast cancer. $\mathrm{N}$ Engl J Med 2012;367:1783-1791. 